\title{
Photoinduced copper-catalyzed asymmetric C-O
}

\section{cross-coupling}

Jun Chen ${ }^{1,3}$, Yu-Jie Liang ${ }^{2,3}$, Peng-Zi Wang ${ }^{1}$, Guo-Qing Li $^{1}$, Bin Zhang ${ }^{1}$, Hao Qian ${ }^{1}$, Xiao-Die Huan ${ }^{1}$, Wei Guan $^{2, *}$, Wen-Jing Xiao ${ }^{1, *}$ and Jia-Rong Chen ${ }^{1, *}$

${ }^{1}$ CCNU-uOttawa Joint Research Centre, Key Laboratory of Pesticides \& Chemical Biology Ministry of Education, College of Chemistry, Central China Normal University, 152 Luoyu Road, Wuhan, Hubei 430079, People's Republic of China

${ }^{2}$ Institute of Functional Material Chemistry, Faculty of Chemistry, Northeast Normal University, Changchun 130024, People's Republic of China

${ }^{3}$ These authors contributed equally: Jun Chen, Yu-Jie Liang.

*guanw580@nenu.edu.cn; wxiao@mail.ccnu.edu.cn; chenjiarong@mail.ccnu.edu.cn

Construction of carbon-heteroatom bonds is one of the most active areas of research in organic chemistry, because the function of organic molecules is often derived from the presence of heteroatoms. Whereas considerable advances have recently been achieved in radical-involved catalytic asymmetric $\mathrm{C}-\mathrm{N}$ bond formation, there has been little progress in the corresponding $\mathrm{C}-\mathrm{O}$ bond-forming processes. Here we describe a photoinduced copper-catalyzed cross-coupling of readily oxime esters and 1,3-dienes to generate diversely substituted allylic esters with high regio- and enantioselectivity ( $>75$ examples; up to $95 \%$ ee). The reaction proceeds at room temperature under excitation by purple light-emitting diodes and features the use of a single, earth-abundant copper-based chiral catalyst as both the photoredox catalyst for radical generation and the source of asymmetric induction in C-O coupling. Combined experimental and DFT computational studies suggest the formation of $\pi$-allylcopper complex from redox-active oxime esters as bifunctional reagents and 1,3-dienes through a radical-polar crossover process.

The scaffold of organic compounds mainly consists of carbon-carbon bonds, but their functions are often derived from the presence of various heteroatoms. In medicinal chemists' toolbox, the assembly of carbon-heteroatom bonds accounts for almost half of all top ten major categories of chemical reactions employed in the drug discovery ${ }^{1,2}$. Not surprisingly, the development of more efficient and sustainable methods for the construction of carbon-heteroatom bonds, particularly the $\mathrm{C}-\mathrm{N}$ and $\mathrm{C}-\mathrm{O}$ bonds, continues to attract considerable modern synthetic efforts. With the evolvement of radical chemistry and transition-metal catalysis, radical-involved cross-coupling are emerging as a particularly straightforward strategy for construction of various carbon-heteroatom bonds ${ }^{3-6}$. The remarkable findings of $\mathrm{Fu}$ and Peters demonstrate that photoinduced asymmetric copper catalysis provides a robust and reliable method for enantioselective $\mathrm{C}-\mathrm{N}$ coupling of racemic tertiary alkyl chlorides with amines under mild conditions (Fig. 1) $)^{7,8}$. Subsequent works of $\mathrm{Liu}^{9-11}$ and $\mathrm{Liu}^{12}$ showed that certain chiral ligands (phosphoric acid, bisoxazolines) and directing groups substantially improved the copper's catalytic activity, and opened up a much broader substrate scope of radical precursors and $N$-nucleophiles. Notably, MacMillan's $\mathrm{s}^{13}$ organocatalytic photoredox-based approach, Zhang's Co(II)-based metalloradical catalysis ${ }^{14}$ and Nagib's ${ }^{15}$ 
radical relay chaperone strategy also provided a range of complementary strategies for direct enantioselective $\mathrm{N}$-centered radical-mediated $\mathrm{sp}^{3} \mathrm{C}-\mathrm{N}$ bond formation. Despite these considerable advances, there has been little progress in corresponding radical-involved $\mathrm{C}-\mathrm{O}$ bond-forming processes. With several recent noteworthy exceptions of alkene-based intramolecular enantioselective C-O bond-forming radical cylizations from the groups of Buchwald ${ }^{16,17}$, and $\mathrm{Liu}^{18}$, the development of new catalytic systems for radical-involved catalytic asymmetric intermolecular C-O coupling remains an unsolved and long-standing challenge ${ }^{19,20}$.

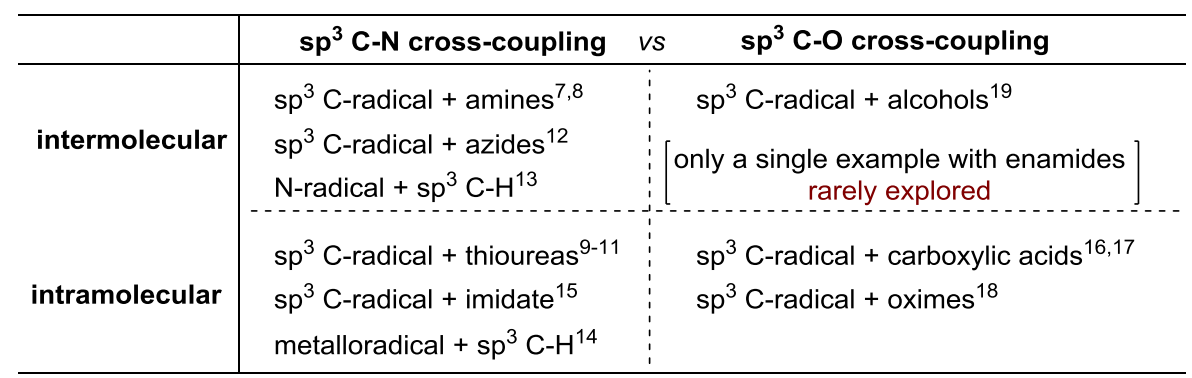

Fig. 1 | Qualitative overview on the radical-involved $\mathrm{sp}^{3} \mathrm{C}-\mathrm{N}$ and $\mathrm{sp}^{3} \mathrm{C}-\mathrm{O}$ cross-coupling.

The copper-catalyzed asymmetric allylic substitution provides a less expensive and potentially equally efficient approach for construction of valuable enantiopure allylic compounds compared to the dominant $\mathrm{Pd}, \mathrm{Rh}$, and Ir-based catalytic systems ${ }^{21,22}$. The key intermediates-the $[\sigma+\pi]$-allyl copper(III) or $\pi$-allyl copper(III) complexes-are mostly formed from nucleophilic organometallic species and electrophilic allylic systems having a leaving group through ionic mechanisms (Fig. 2a) ${ }^{23}$. Despite powerful, however, the use of highly reactive and moisture sensitive reagents (e.g., organomagnesium, organolithium, organoaluminum, organozinc, or the milder organoboron reagents) and prefunctionalized allylic systems considerably limited the substrate range. Notably, application of this strategy to the heteroatom nucleophiles as coupling partners has not been realized in copper-catalyzed asymmetric allylic alkylation. Recent advances in copper-catalyzed photoredox and radical chemistry have shown that photorexcited copper catalysts can not only generate highly reactive radical species from various redox-active precursors but also trap these open-shell species to enable ensuing asymmetric coupling ${ }^{24-26}$. Liu has shown that copper(II)-bound $\mathrm{N}$-centered radical could cleavage the allylic $\mathrm{sp}^{3} \mathrm{C}-\mathrm{H}$ bond of alkenes in a site-specific manner via hydrogen atom transfer to generate allylic radicals ${ }^{27}$. This method allows site- and enantioselective allylic C-H cyanation. Quite recently, the groups of Glorius ${ }^{28-32}$, Gevorgyan ${ }^{33}$, and $\mathrm{Shi}^{34}$ demonstrated that alkyl radicals, which are generated from $N$-hydroxyphthalimide esters or alkyl halides under photoredox catalysis, can be sequentially trapped by 1,3-dienes and palladium, chromium, or titanium catalyst to generate the corresponding electrophilic or nucleophilic $\pi$-allylmetal complexes. Employing these intermediates, they achieved a range of aminoalkylation and dialkylation reactions of 1,3-dienes. However, in most of these transformations, the control of both regio- and stereoselectivity of these radical transformations remains a formidable challenge. Drawing inspiration from these breakthroughs and based on our long standing interest in photoinduced copper catalysis ${ }^{35}$, we questioned whether $\pi$-allylcopper complexes could also be formed by a radical pathway to surmount the aforementioned limitations often encountered in traditional copper-catalyzed ionic asymmetric allylic substitution (Fig. 2b). Specifically, we hypothesized that a photoexcited $\mathrm{Cu}(\mathrm{I})$ complex might reduce a redox-active radical precursor via a single electron transfer (SET) process to generate a radical species and a $\mathrm{Cu}(\mathrm{II})$ species. Then, interception of such a radical species by a feedstock 1,3-diene would give a new allyl radical that might be readily trapped by the initially formed $\mathrm{Cu}(\mathrm{II})$ species to generate 
$\pi$-allylcopper(III)/nucleophile complex in the presence of a nucleophilic reagent. Finally, this high-valent $\mathrm{Cu}$ (III) species is prone to undergo reductive elimination to form a new $\mathrm{C}-\mathrm{O}$ chemical bond with concomitant formation of $\mathrm{Cu}(\mathrm{I})$ catalyst $^{36,37}$. To date, exploration of this strategy in the development of an asymmetric allylic $\mathrm{sp}^{3} \mathrm{C}-\mathrm{O}$ coupling, particularly the intermolecular variant, has not yet been documented ${ }^{38-40}$.

Given their readily availability from ketones and carboxylic acids as well as susceptibility to SET reduction, oxime esters have been widely used as cyanoalkyl radical precursors in various radical cross-coupling reactions ${ }^{41-43}$. Based on our design plan (Fig. 2b), we hypothesized that $\pi$-allylcopper(III) complex might be generated by combining oxime esters and 1,3-dienes under photoinduced copper catalysis. However, at the outset of this study, we were not sure oxime esters can serve as bifunctional reagents-as both radical sources and $O$-nucleophile, or whether the regio- and stereocontrol can be realized by using a chiral ligand in such a radical-polar crossover process ${ }^{44,45}$. Here we describe a radical strategy for formation of $\pi$-allylcopper(III) complex and achievement of its highly regio- and enantioselective asymmetric C-O cross-coupling under photoindcued copper catalysis (Fig. 2c). The protocol lies at the intersection of several significant fields of modern chemical catalysis. The obtained enantioenriched 1,2-cyanoalkylesterification products of 1,3-dienes are important building blocks in both synthetic and medicinal chemistry.
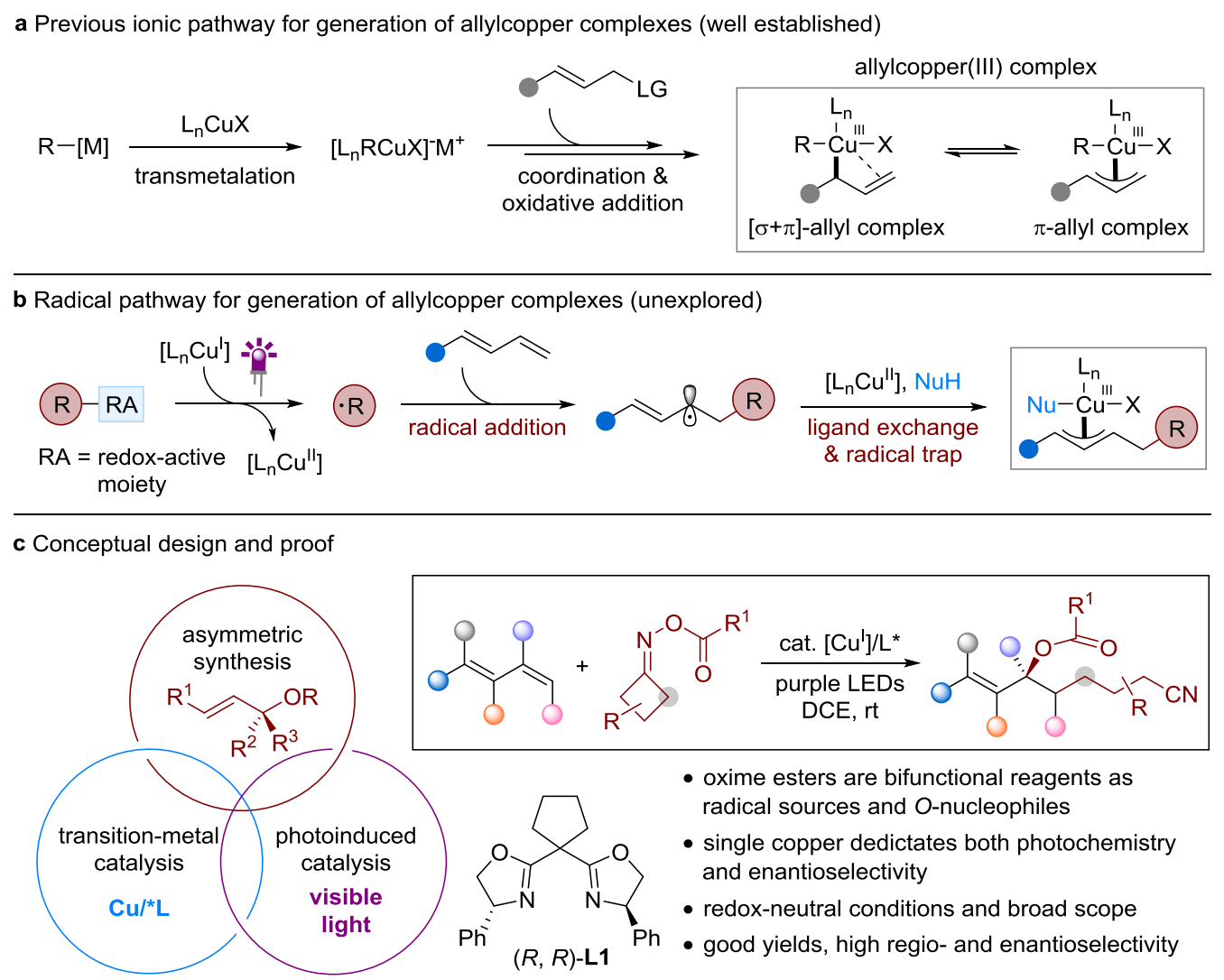

Fig. 2 | Strategic development for formation of $\pi$-allylcopper complexes and new reaction design. a, Previous thermal ionic pathway. $\mathbf{b}$, Outline of a radical pathway (unexplored). $\mathbf{c}$, Conceptual design and proof (this work, catalytic asymmetric $\mathrm{sp}^{3} \mathrm{C}-\mathrm{O}$ coupling enabled by a radical formation of $\pi$-allylcopper complexes). This work features the use of a single chiral copper catalyst both as photo- and coupling catalysts and oxime esters as bifunctional reagents. This protocol enables efficient incorporation of cyanoalkyl moiety and $\mathrm{sp}^{3} \mathrm{C}-\mathrm{O}$ bond formation with good functional-group tolerance and excellent regio- 
and enantioselectivity, leading to many valuable chiral allylic esters (>75 examples).

\section{Results and discussion}

Reaction development and optimization. On the basis of our conceptual design, we began the study by examining cross-coupling reaction of 1-phenylbutadiene $1 \mathbf{a}$ and oxime ester $\mathbf{2} \mathbf{a}$ under visible-light-induced copper catalysis (Table 1). The major challenge was to control both regio- (1,2-vs 1,4-adduct) and enantioselectivity. After extensive screening of reaction parameters, we discovered that irradiation of the cross-coupling partners with purple LEDs at room temperature for 2 hours in the presence of $\mathrm{Cu}\left(\mathrm{CH}_{3} \mathrm{CN}\right)_{4} \mathrm{PF}_{6}(1.0 \mathrm{~mol} \%)$ and a chiral bisoxazoline ligand $\mathbf{L 1}(1.2 \mathrm{~mol} \%)$ gave the best results, affording the desired 1,2-adduct 3aa in $79 \%$ isolated yield with $94 \%$ enantiomeric excess (ee) (entry 1) (Supplementary Tables S1-5). Notably, the competitive side product 1,4-adduct 4aa was not detected. The reaction efficiency appeared to be sensitive to the solvents and copper salts. For instance, the use of THF and DMF led to significant decrease of yield (entries 2 and 3). While employment of $\mathrm{Cu}(\mathrm{OTf})_{2}$ gave comparable results, the reaction did not work when using Cul, with 2a being fully recovered (entries 4 and 5). The substitution patterns of the ligands have also played an essential role. Compared to ligand L1, ligands L2-L4 resulted in substantially lower reaction efficiency and enantioselectivity (entries 6-8). Interestingly, the reaction that was performed under irradiation of blue LEDs $(\lambda \max =460 \mathrm{~nm})$ proceeded sluggishly, with 3aa being obtained in only $5 \%$ yield while with $91 \%$ ee (entry 9 ).

Table 1 | Effect of variations in the reaction parameters and conditions

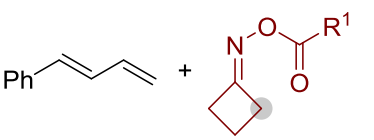

$1 \mathbf{a}$

2a $\left(\mathrm{R}^{1}=p-\mathrm{MeC}_{6} \mathrm{H}_{4}\right)$

( 1.5 equiv.)

$(0.2 \mathrm{mmol})$
$\mathrm{Cu}\left(\mathrm{CH}_{3} \mathrm{CN}\right)_{4} \mathrm{PF}_{6}(1.0 \mathrm{~mol} \%)$

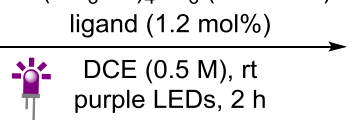

purple LEDs, $2 \mathrm{~h}$

"standard conditions"

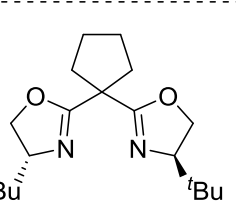

$(R, R)$-L2

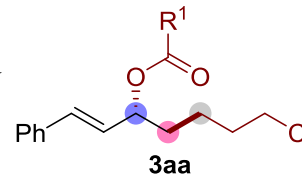

(1,2-adduct)

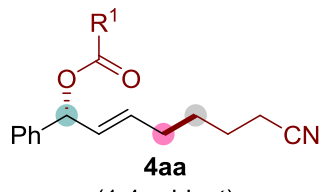

(1,4-adduct)

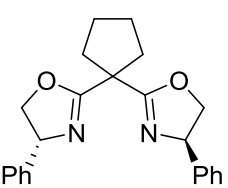

$(R, R)$-L1

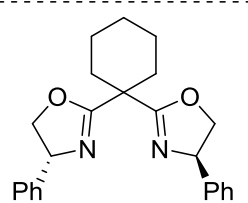

$(R, R)$-L3

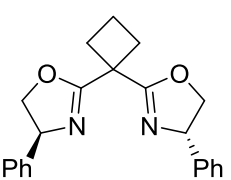

$(S, S)$-L4

\begin{tabular}{|c|c|c|c|}
\hline Entry & Variation $^{\mathrm{a}}$ & Yield $(\%)^{\mathrm{b}}$ & ee $(\%)^{\mathrm{c}}$ \\
\hline 1 & None & $88(79)^{\mathrm{d}}$ & 94 \\
\hline 2 & Use of THF, $16 \mathrm{~h}$ & $21(33)^{\mathrm{e}}$ & 86 \\
\hline 3 & Use of DMF, $16 \mathrm{~h}$ & $0(99)^{\mathrm{e}}$ & - \\
\hline 4 & Use of $\mathrm{CuI}$ & $0(99)^{\mathrm{e}}$ & - \\
\hline 5 & Use of $\mathrm{Cu}(\mathrm{OTf})_{2}$ & 81 & 94 \\
\hline 6 & Ligand $(R, R)-\mathbf{L} 2$ & $15(16)^{\mathrm{e}}$ & 9 \\
\hline 7 & Ligand $(R, R)-\mathbf{L} 3$ & 61 & 88 \\
\hline 8 & Ligand $(S, \mathrm{~S})-\mathbf{L 4}$ & 69 & -82 \\
\hline 9 & Blue LEDs & $5(63)^{\mathrm{e}}$ & 91 \\
\hline 10 & No light & $0(99)^{\mathrm{e}}$ & - \\
\hline 11 & No ligand $(R, R)-\mathbf{L 1}$ & $0(22)^{\mathrm{e}}$ & - \\
\hline 12 & $\mathrm{No} \mathrm{Cu}\left(\mathrm{CH}_{3} \mathrm{CN}\right)_{4} \mathrm{PF}_{6}$ & $0(99)^{\mathrm{e}}$ & - \\
\hline 13 & Added water ( 0.1 equiv.) & 84 & 94 \\
\hline
\end{tabular}

${ }^{a}$ Reaction conditions: 1a $(0.3 \mathrm{mmol}), \quad 2 a(0.2 \mathrm{mmol}), \mathrm{Cu}\left(\mathrm{CH}_{3} \mathrm{CN}_{4}\right)_{4} \mathrm{PF}_{6}(1.0 \mathrm{~mol} \%)$, ligand $\mathbf{L 1}(1.2 \mathrm{~mol} \%)$, 1,2-dichloroethane (DCE) $(4.0 \mathrm{~mL}), 2$ x $20 \mathrm{~W}$ purple LEDs $(\lambda \max =390 \mathrm{~nm})$, at room temperature. ${ }^{\mathrm{b}}$ Yields were determined by GC analysis with 1,3,5-trimethoxybenzene as the internal standard. ${ }^{c}$ Determined by HPLC analysis on a chiral stationary phase. ${ }^{\mathrm{d}}$ Isolated yield. ${ }^{\mathrm{e}}$ Recovery of $\mathbf{2 a}$.

Control experiments established that each component is critical to the desired cross-coupling reaction 
(entries 10-12). Remarkably, without ligand L1, the majority of oxime ester 2a was consumed, but only leading to a messy mixture. Our concern that allylic radical initially formed upon radical addition might isomerize to oxidation labile benzylic radical to lead to 1,4-adduct appears to be unfounded, as evidenced by our observation of exclusive formation of 1,2-adduct. From a practical point of view, it is worthy of noting that $\mathrm{Cu}\left(\mathrm{CH}_{3} \mathrm{CN}\right)_{4} \mathrm{PF}_{6}$ and chiral bisoxazoline ligand are commercially available and the protocol is not moisture-sensitive (entry 13). Until now, the oxime esters have been mainly used a source of cyanoalkyl radical source with generation of a stoichiometric carboxylic anion as byproduct. By contrast, this protocol allows the waste carboxylic anions to be used as $O$-nucleophiles, leading to atom-economic incorporation of the oxime esters.

Reaction scope. With the optimized conditions in hand, we examined the sbustrate scope with respect to the two coupling partners on a $0.2 \mathrm{mmol}$ scale. First, the scope of oxime esters was tested by variation of the $\mathbf{O}$-acyl moiety (Table 2a). Aside from $\mathbf{2 a}$, aryl carboxylic acid-derived oxime esters $\mathbf{2} \mathbf{b}$ - $\mathbf{h}$ bearing various netural $(\mathrm{H})$, electron-donating (e.g., $\mathrm{Me}, t-\mathrm{Bu}, \mathrm{OMe}$ ) or electron-withdrawing (e.g., $\mathrm{Cl}, \mathrm{Br}, \mathrm{I}, \mathrm{CO}_{2} \mathrm{Me}$ ) groups at the para-position of the aromatic ring are all capaple partners for the cross-coupling. The desired products 3ab-ah were obtained in generally good yields (62-78\%) with excellent enantioselectivity (91-93\% ee). Notably, the halogen atoms $(\mathrm{Cl}, \mathrm{Br}, \mathrm{l})$ may be subjected to further synthetic manipulations (in particular transition metal-catalyzed cross-coupling reactions) at the halogenated positions (products 3ae-ag). Moreover, the reaction of oxime esters 1i-k with functional groups at the meta- or ortho-position also reacted well, affording 3ai-ak in $73-80 \%$ yields with $91-94 \%$ ee, indicating that the steric hindrance did not affect the transformation. Reactions with substrates having a fused aromatic ring (2I) or heteroaromatic ring $(\mathbf{2} \mathbf{m}-\mathbf{p})$ were also amenable to this process, giving the corresponding coupled products 3al-ap in good yields with consistently excellent enanitoselectivity (89-94\% ee). Then, we proceeded to examine the generality of this asymmetric cross-coupling by using a range of aliphatic carboxylic acid-derived oxime esters. For instance, oxime esters $\mathbf{2} \mathbf{q}-\mathbf{w}$ derived from cyclobutanone and primary, secondary (cyclohexyl), tertiary acids (e.g., $t$-butyl and adamantyl acids) as well as $\alpha, \beta$-unsaturated acids were all well accomodated under the current catalytic system. Thus, the expected allylic ester products 3aq-aw were isolated in good yields ranging from $54 \%$ to $94 \%$ with $90-94 \%$ ee.

Markedly, as shown in Table 2b, oxime esters $\mathbf{2 x - z}$, and $\mathbf{2 a a}$ that are derived from drugs Diclofenac, Febuxostat, Gemfibrozil, and Ciprofibrate were also converted into their corresponding 1,2-cyanoalkylacyloxylation products 3ax, 3ay, 3az, and 3aaa with good yields and satisfactory enantioelectivity, respectively. Naturally occurring acid and amino acid derived oxime esters 2 ab-ac were also suitable for the coupling, providing the expected products with good yields and stereoselectivity. These results showed the robustness and utility of the developed reaction.

Finally, we turned our attention to investigating the effect of variation of oxime moiety on the reaction efficiency and enantioselectivity by reacting with 1a (Table 2c). The reaction of 3,3-disubstituted oxime esters $\mathbf{5 a}$ and $\mathbf{5 b}$ proceeded smoothly to afford the expected products $\mathbf{6 a a}$ and $\mathbf{6 a b}$ in high yields with $93 \%$ and $92 \%$ ee, respectively. Sterically more demanding $5 \mathrm{c}$ can also be well tolerated to give 6ac with modest results. 2-Methyl substituted oxime ester $\mathbf{5} \mathbf{d}$ also participated in the reaction efficiently to produce a good yield of 6ad with high ee values, but with 1:1 d.r. caused by initial non-enantioselective addition of cyanoalkyl radical. Notably, benzocyclobutenone-derived oxime ester $5 e$ also proved to be suitable for the reaction, leading to product 6 ae in $75 \%$ yield with $92 \%$ ee.

Table 2 | Scope of the oxime esters in photoinduced copper-catalyzed asymmetric C-O cross-coupling ${ }^{a}$ 

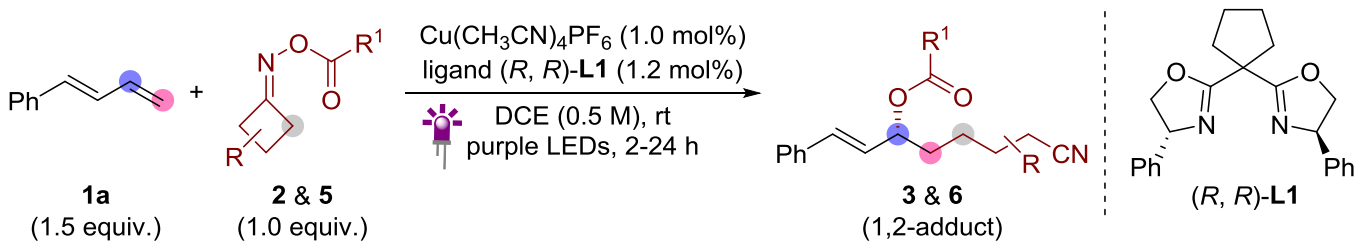

$(R, R)-\mathrm{L} 1$

(1.5 equiv.) (1.0 equiv.)

a variation of $O$-acyl moiety

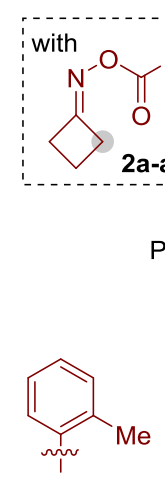

3ak

\section{(1)}<smiles>N#CCCCCC(C=Cc1ccccc1)OC=O</smiles>

R 3aa, $\mathrm{R}=\mathrm{Me}$, 3ab, $\mathrm{R}=\mathrm{H}$,

3ac, $\mathrm{R}=t-\mathrm{Bu}$

3ad, $\mathrm{R}=\mathrm{OMe}$,

3ae, $\mathrm{R}=\mathrm{Cl}$,

3af, $\mathrm{R}=\mathrm{Br}$

3ag, $\mathrm{R}=\mathrm{I}$,

$79 \%, 94 \%$ ee

$71 \%, 93 \%$ ee

$62 \%, 93 \%$ ee

$65 \%, 92 \%$ ee

$77 \%, 92 \%$ ee

$78 \%, 92 \%$ ee

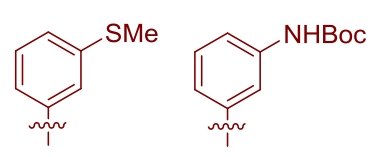

3ai

3aj

$73 \%, 92 \%$ ee $78 \%, 91 \%$ ee

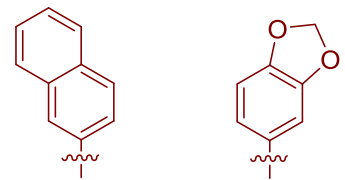

3am

$3 a l$

$80 \%, 94 \%$ ee $\quad 73 \%, 91 \%$ ee $\quad 75 \%, 92 \%$ ee $\quad 70 \%, 94 \%$ ee $\quad 80 \%, 90 \%$ ee $\quad 62 \%, 89 \%$ ee

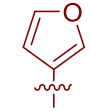

3an

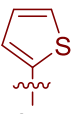

3ao

(2)
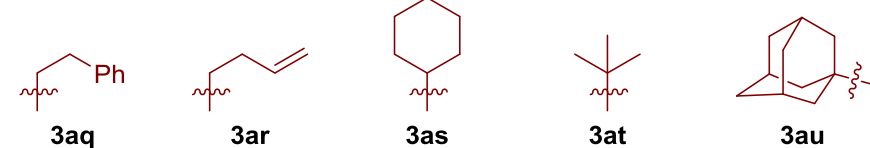

3au

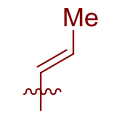

3av

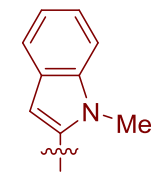

3ap

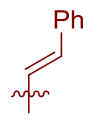

3aw

$71 \%, 90 \%$ ee $\quad 78 \%, 90 \%$ ee $\quad 94 \%, 93 \%$ ee $\quad 85 \%, 94 \%$ ee $\quad 88 \%, 93 \%$ ee $\quad 68 \%, 94 \%$ ee $\quad 54 \%, 91 \%$ ee

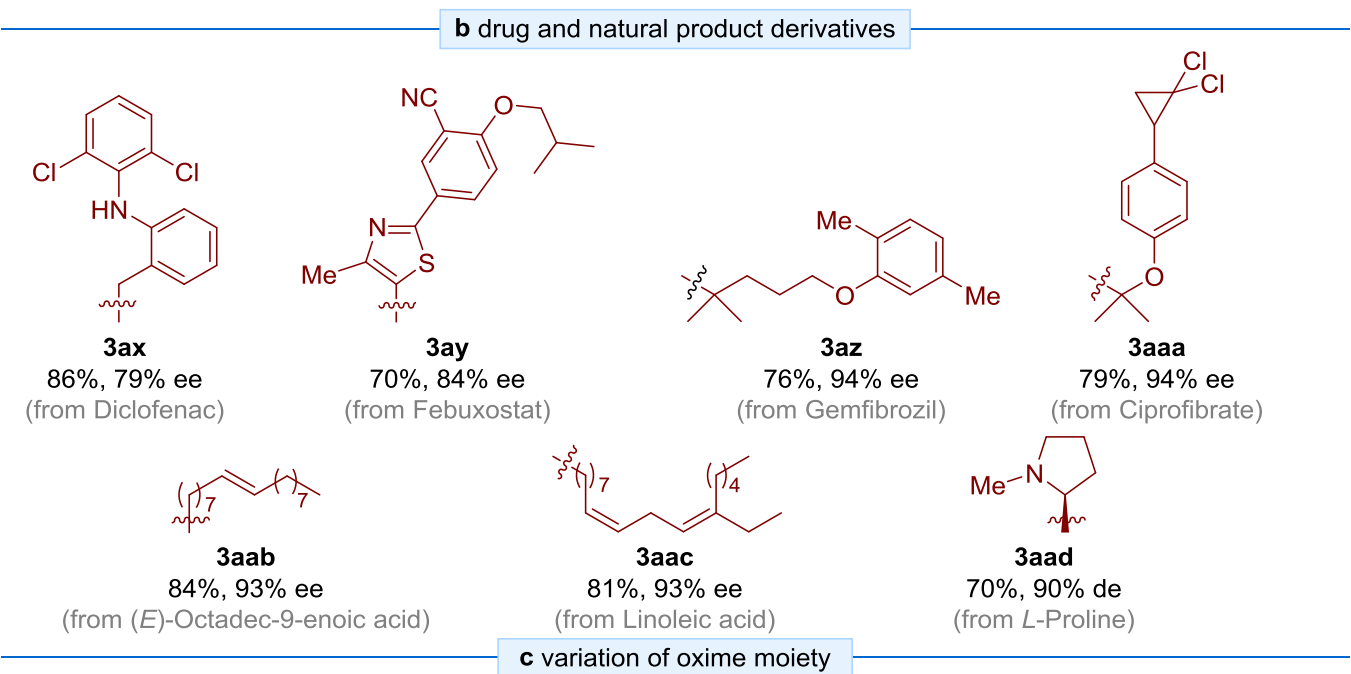

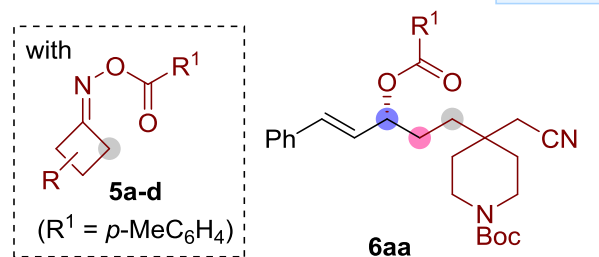

$87 \%, 93 \%$ ee

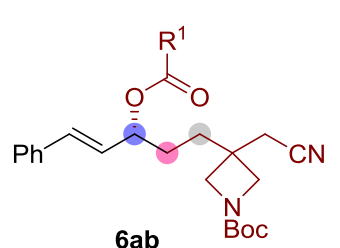

$91 \%, 92 \%$ ee<smiles>[R1]C(=O)OC(C=Cc1ccccc1)CC(C)(C)C(=O)C(C)(C)C#N</smiles>

6ac, $78 \%{ }^{\mathrm{b}}$

$85 \%$ ee $(E / Z=17: 1)$<smiles>[R]C(=O)O[Ga](/C=C/c1ccccc1)CC(C)CCC#N</smiles>

6ad, $70 \%(1: 1 \text { d.r. })^{b}$ $93 \%$ ee, $94 \%$ ee

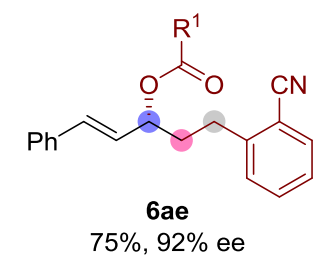

${ }^{a}$ Reaction conditions: 1a $(0.3 \mathrm{mmol}), 2(0.2 \mathrm{mmol}), \quad \mathrm{Cu}\left(\mathrm{CH}_{3} \mathrm{CN}\right)_{4} \mathrm{PF}_{6}(1.0 \mathrm{~mol} \%)$, ligand $\mathbf{L 1}(1.2 \mathrm{~mol} \%)$, 
1,2-dichloroethane (DCE) $(4.0 \mathrm{~mL}), 2 \times 20 \mathrm{~W}$ purple LEDs $(\lambda \max =390 \mathrm{~nm})$, at room temperature; isolated yields. ${ }^{b}$ With $\mathrm{Cu}\left(\mathrm{CH}_{3} \mathrm{CN}\right)_{4} \mathrm{PF}_{6}(2.0 \mathrm{~mol} \%)$, ligand $\mathbf{L 1}(2.4 \mathrm{~mol} \%)$, and 3.0 equiv. of $\mathbf{1 a}$.

Next, a representative set of 1,3-dienes were evaluated to further investigate the generality of this cross-coupling (Table 3). First, it was found that the 1-aryl and heteroaryl substituted 1,3-dienes were all competent reaction partners. For example, substrates $\mathbf{1} \mathbf{b}$-g bearing either alkyl or electron-withdrawing groups (e.g., $\mathrm{F}, \mathrm{Cl}, \mathrm{Br}, \mathrm{CF}_{3}$ ) at the para-position of the phenyl ring were well tolerated, furnishing the corresponding allylic esters 7 ba-ga in $60-73 \%$ yields with $87-92 \%$ ee. Again, as shown in the reactions of $\mathbf{l} \mathbf{h}-\mathrm{m}$, the variation of the substitution pattern and steric hindrance of the aromatic ring has little effect on both the reaction efficiency and enantioselectivity. As such, the expected products 7 ha-ma were isolated in $70-80 \%$ yields with $87-95 \%$ ee, regardless of the electronic properties of the substituent. The reaction of 1-(2-naphthyl)-substituted 1,3-diene $1 \mathrm{n}$ also proceeded smoothly to produce $\mathbf{7 n a}$ in $53 \%$ yield with $88 \%$ ee. Additionally, 1,3-dienes 10 and $1 \mathrm{p}$ with 3-furanyl or 3-thiophenyl were well tolerated, furnishing the relative products $70 a$ and $7 \mathrm{pa}$ in good yields with $88 \%$ and $91 \%$ ee, respectively. Notably, as shown in the cases of 1q-s, 1,1-disubstituted 1,3-dienes and those bearing a methyl group at C2 or C4 position were also proven to be suitable coupling partners. The expected products $7 \mathrm{ra}$ and $7 \mathrm{sa}$ were obtained with good yields and satisfactory enantioselectivity, while 7qa was isolated with moderate yield due to low conversion. The 1:1 d.r. of 7sa was caused by the initial non-enantioselective radical addition of the cyanoalkyl radical.

Challenging alkyl-substituted 1,3-dienes such as $\mathbf{1 t}$ and $\mathbf{1 u}$ could also participate in the desired cross-coupling. In both cases, however, mixtures of 1,2- and 1,4-adducts were isolated in good yields but with moderate regio- and enantioselectivity, probably because of the sterically similarity at C-2 and C-4 positions of such type of 1,3-dienes. We are currently investigating modifications to the chiral ligand, which will provide better sterocontrol for these alkyl-substituted 1,3-dienes. X-ray crystallographic analysis of product $\mathbf{7 f h}$, produced in the reaction of oxime ester $\mathbf{1 f}$ and $\mathbf{2} \mathbf{h}$ with $\mathbf{7 7 \%}$ yield and $\mathbf{9 2 \%}$ ee, confirmed the $(R)$-configuration of the newly formed absolute stereochemistry. Thus, all other cross-coupling products were tentatively assigned by analogy with $\mathbf{7 f h}$. Remarkably, biologically relevant molecule, Febuxostat-derived 1,3-diene $\mathbf{1} \mathbf{v}$, could also couple with $\mathbf{2 a}$ efficiently to give the desired product 7 va in $52 \%$ yield with $84 \%$ ee.

Table 3 | Scope of 1,3-dienes in photoinduced copper-catalyzed asymmetric C-O cross-coupling ${ }^{\mathrm{a}}$ 

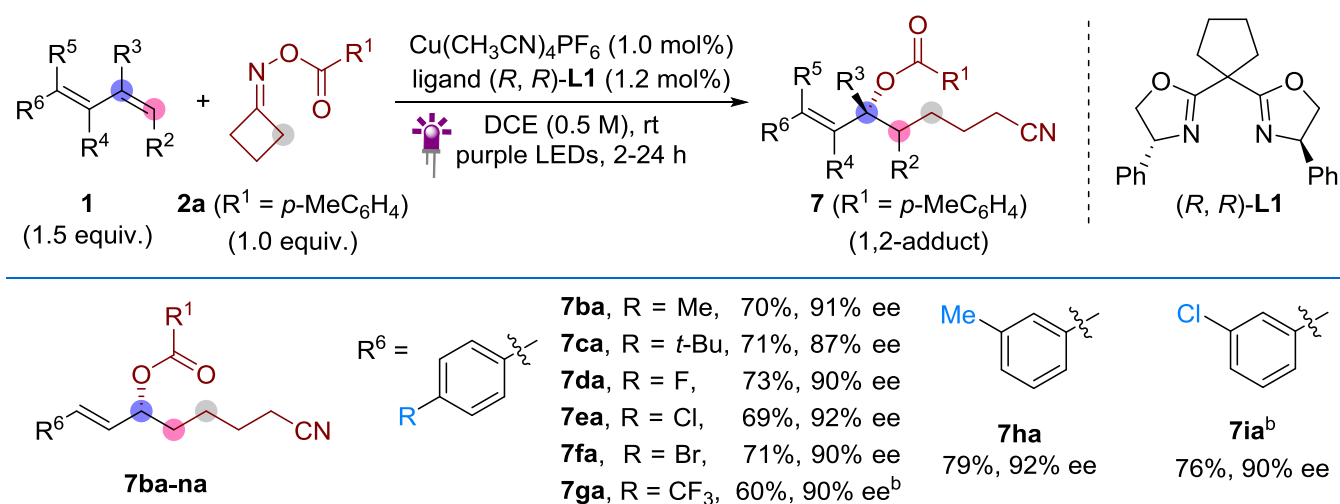

7ba, $\mathrm{R}=\mathrm{Me}, \quad 70 \%, 91 \%$ ee $7 \mathrm{ca}, \mathrm{R}=t-\mathrm{Bu}, 71 \%, 87 \%$ ee 7 da, $R=F, \quad 73 \%, 90 \%$ ee 7ea, $\mathrm{R}=\mathrm{Cl}, \quad 69 \%, 92 \%$ ee $7 \mathrm{fa}, \mathrm{R}=\mathrm{Br}, \quad 71 \%, 90 \%$ ee 7 ga, $\mathrm{R}=\mathrm{CF}_{3}, 60 \%, 90 \% \mathrm{ee}^{\mathrm{b}}$<smiles>Cc1cccc(I)c1</smiles>

7ha

$79 \%, 92 \%$ ee<smiles>Cc1cccc(Cl)c1</smiles>

$7 \mathbf{i a}^{\mathrm{b}}$

$76 \%, 90 \%$ ee<smiles>Fc1cccc(I)c1</smiles>

$7 \mathbf{j a}^{\mathrm{b}}$<smiles>Cc1ccccc1C</smiles>

$71 a^{b}$<smiles>Cc1ccccc1Cl</smiles>

$7 \mathrm{ma}^{\mathrm{b}}$<smiles>Ic1ccc2ccccc2c1</smiles>

$7 \mathrm{na}^{\mathrm{b}}$<smiles>CC(C)(C)c1ccoc1</smiles>

$70 a^{\mathrm{b}}$

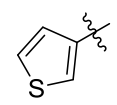

$7 \mathrm{pa}^{\mathrm{b}}$

$73 \%, 93 \%$ ee $\quad 80 \%, 89 \%$ ee $\quad 70 \%, 95 \%$ ee $\quad 80 \%, 87 \%$ ee $\quad 53 \%, 88 \%$ ee $\quad 65 \%, 88 \%$ ee $\quad 71 \%, 91 \%$ ee

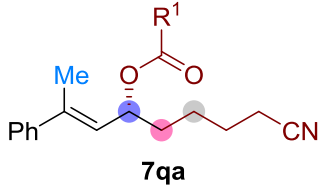

$24 \%, 49 \% e^{b, c}$<smiles>[R]C(=O)O[C@@H](C=CCCC#N)C(=C)O[N+](=O)c1ccccc1</smiles>

$7 \mathrm{ra}, 78 \%$

$95 \%$ ee $(E / Z=20: 1)^{b}$<smiles>[R]C(=O)O[C@@H](/C=C/c1ccccc1)C(=O)O</smiles>

7 sa, $76 \%$ (1:1 d.r.)

$88 \%$ ee, $85 \%$ ee ed $^{\text {, d }}$

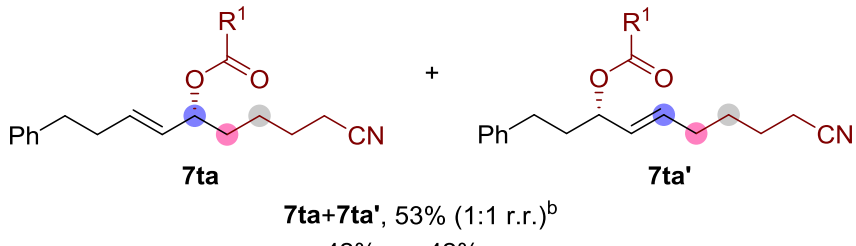

$42 \%$ ee, $42 \%$ ee

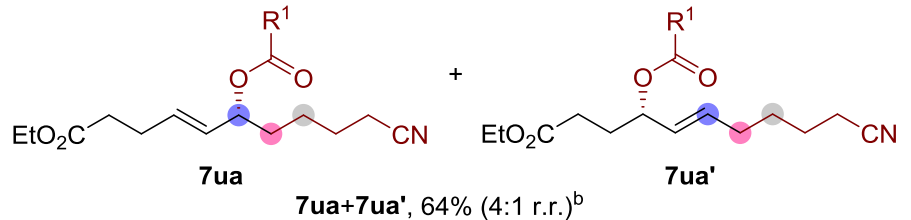

$17 \%$ ee, $58 \%$ ee $\mathrm{CO}_{2} \mathrm{Me}$

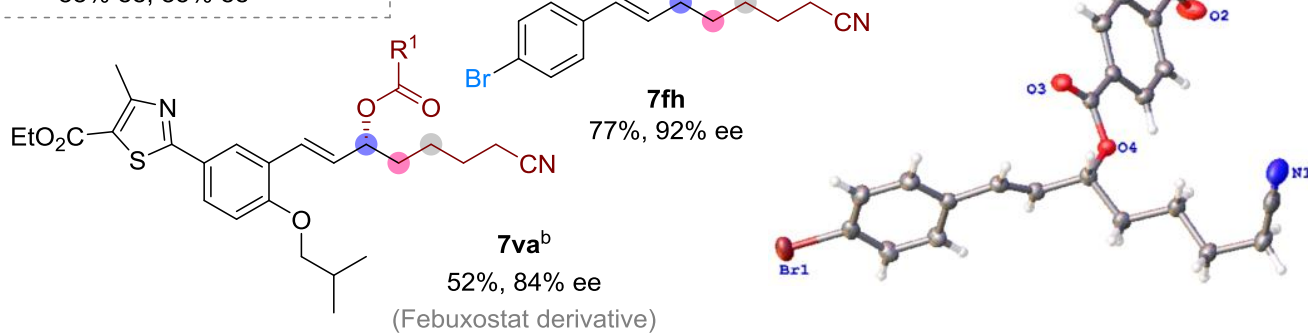

${ }^{a}$ Reaction conditions: $\mathbf{1}(0.3 \mathrm{mmol}), \mathbf{2 a}(0.2 \mathrm{mmol}), \mathbf{C u}\left(\mathrm{CH}_{3} \mathrm{CN}\right)_{4} \mathrm{PF}_{6}(1.0 \mathrm{~mol} \%)$, ligand $\mathbf{L 1}(1.2 \mathrm{~mol} \%)$, 1,2-dichloroethane (DCE) $(4.0 \mathrm{~mL}), 2 \times 20 \mathrm{~W}$ purple LEDs $(\lambda \max =390 \mathrm{~nm})$, at room temperature; isolated yields. ${ }^{b}$ With $\mathrm{Cu}\left(\mathrm{CH}_{3} \mathrm{CN}_{4} \mathrm{PF}_{6}(2.0\right.$ mol\%), ligand $\mathbf{L 1}(2.4 \mathrm{~mol} \%)$, and 3.0 equiv. of $\mathbf{1} .^{\mathrm{c}}(E) /(Z)-\mathbf{1 q}=10: 1 .{ }^{\mathrm{d}}(E) /(Z)-\mathbf{1 s}=3: 1$.

According to our design plan, an allylic radical might be involved during the formation of the $\pi$-allylcopper complex, which was supposed to be formed upon addition of cyanoalkyl radical to 1,3-diene. Thus, we envisioned that if 2-styrenyl substituted cycloketoxime esters could undergo SET-reduction-mediated ring-opening to give allylic radical under the current catalytic system; then an asymmetric $\mathrm{C}-\mathrm{O}$ cross-coupling might also occur between such allyl radical species and the leaving 
group-carboxylic anion. As such, the reaction would provide an atom-economic process for catalytic asymmetric fragmentation/rearrangement of cycloketoxime esters. As highlighted in Table 4, the reaction indeed worked well and the standard catalytic system showed broad substrate scope. A range of aryl carboxylic acid-derived cycloketoxime esters 8a-g with an electron-donating or electron-withdrawing substituent at the para-, meta-, or ortho-position of the phenyl ring all underwent the desired reaction smoothly. The corresponding products 9a-g were obtained with generally good yields (73-90\%) and excellent enantioselectivity (90-93\% ee). Moreover, substrates $\mathbf{8 h}$ and $\mathbf{8 i}$ containing (hetero)arene annulated systems and $\mathbf{8 j}$ with 2-thiophenyl group were also suitable for the reaction, with the relative products $\mathbf{9 h - j}$ being formed with comparable results. As shown in the synthesis of $\mathbf{9 j - n}$, primary (8k) and secondary (8I), as well as sterically very demanding carboxylic acid-derived cycloketoxime esters (8m and $8 n$ ) can also be well tolerated without loss of reaction efficiency and enantioselectivity (91-93\% ee). We also briefly examined the effect of the $\mathrm{R}^{2}$ group on the reaction. As shown in the reactions of 80-s with $\mathrm{R}^{2}$ being aryl or heteroaryl group, though the change of their electronic properties has some effect on the reaction efficiency, the expected products 90 -s could still be obtained with high to excellent ee values.

Table 4 | Preliminary attempts to achieve photoinduced copper-catalyzed ring-opening asymmetric C-O coupling of cycloketoxime esters ${ }^{a}$
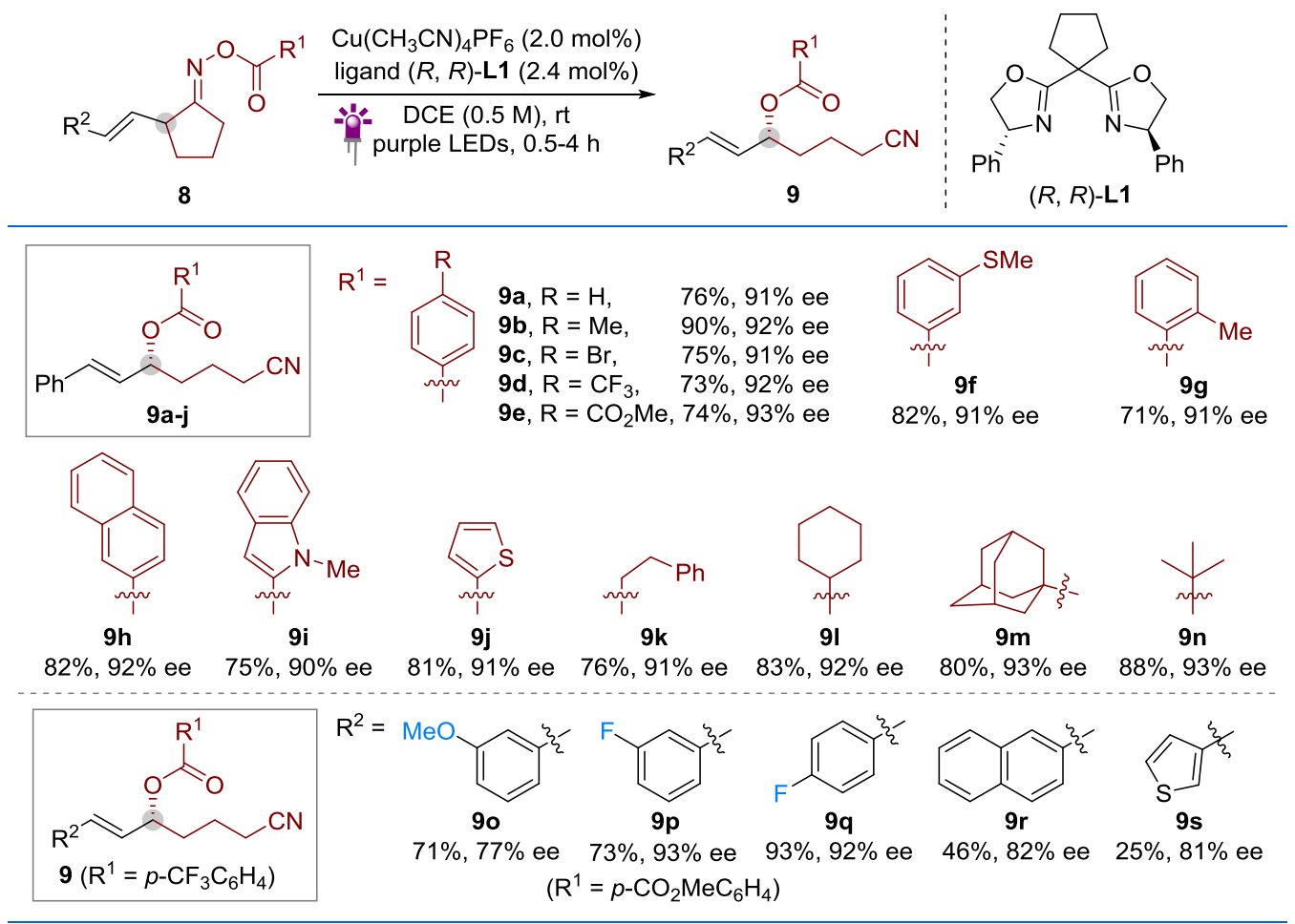

${ }^{\mathrm{a}}$ Reaction conditions: 8 (0.2 mmol), $\mathrm{Cu}\left(\mathrm{CH}_{3} \mathrm{CN}\right)_{4} \mathrm{PF}_{6}(2.0 \mathrm{~mol} \%)$, ligand $\mathbf{L 1}$ (2.4 mol\%), 1,2-dichloroethane (DCE) (4.0 $\mathrm{mL}), 2 \times 20 \mathrm{~W}$ purple LEDs $(\lambda \max =390 \mathrm{~nm})$, at room temperature; isolated yields.

Synthetic applications. A $5.0 \mathrm{mmol}$ scale reaction of $\mathbf{2 a}$ also worked well to give comparable results (3aa, $1.22 \mathrm{~g}, 73 \%$ yield, 93\% ee), indicating the scalability of the process (Fig. 3a). Moreover, the alkene moiety of product 3aa allows further synthetic manipulation. For example, the carbon-carbon double bond in 3aa could undergo hydrogenation easily to give $\mathbf{1 0}$ in good yield without loss of enantioselectivity (Fig. 3b). Irradiation of 3aa with blue LEDs in the presence of photocatalyst $\operatorname{Ir}\left[\mathrm{dF}\left(\mathrm{CF}_{3}\right) \mathrm{ppy}\right]_{2}(\mathrm{dtb}-\mathrm{bpy}) \mathrm{PF}_{6}$ enabled isomerization of $(E)$-3aa to its (Z)-form 11 without a deleterious effect on the enantiopurity (Fig. 3c). 


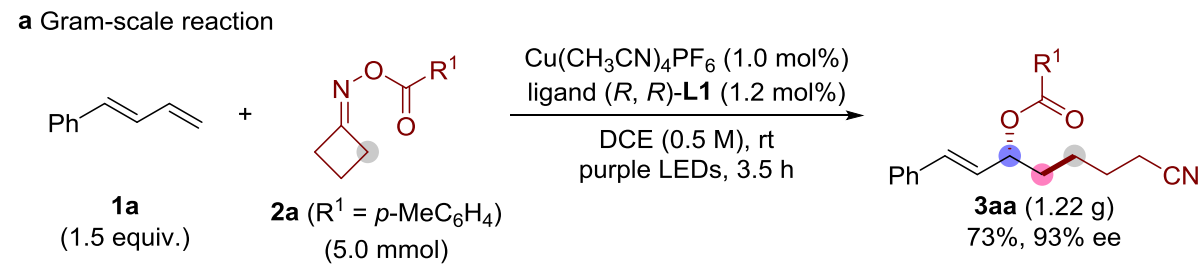

b Reduction of C-C double bond

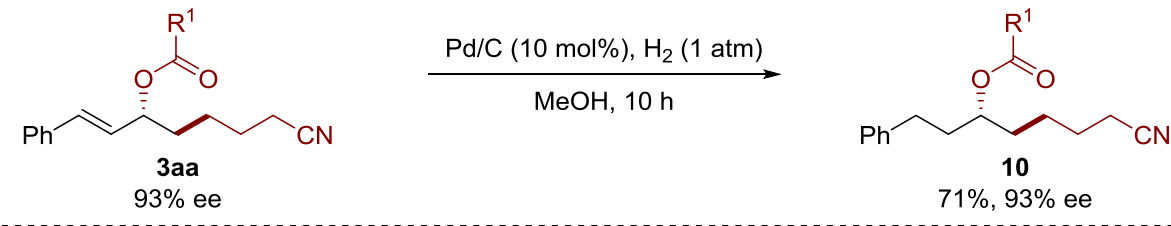

c Photoinduced isomerization of $\mathrm{C}-\mathrm{C}$ double bond
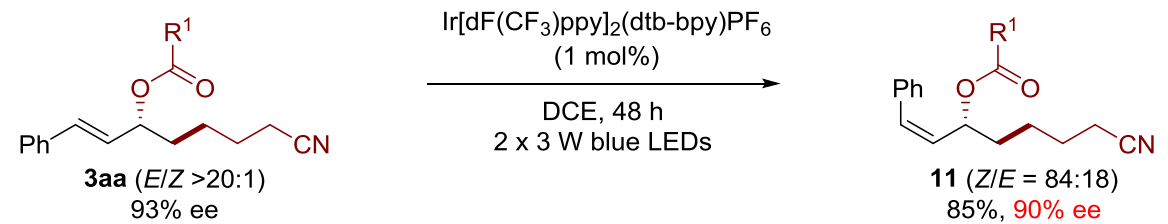

Fig. 3 I Synthetic utility. a, Gram-scale synthetic capability. b, Pd/C-catalyzed reduction of the carbon-carbon double bond. c, Photoinduced isomerization of carbon-carbon double bond.

Mechanistic investigation. First, we obtained UV-Vis absorption spectra of each reaction component, and confirmed that $\mathrm{Cu}\left(\mathrm{CH}_{3} \mathrm{CN}\right)_{4} \mathrm{PF}_{6} / \mathbf{L 1}$ complex was the only visible-light-absorbing species. Stern-Volmer studies of $\mathrm{Cu}\left(\mathrm{CH}_{3} \mathrm{CN}\right)_{4} \mathrm{PF}_{6} / \mathrm{L1}$ with oxime ester 2a supported that a photoexcited $\mathrm{Cu}(\mathrm{I})$ complex could reduce $\mathbf{2} \mathbf{a}$ by SET (see Supplementary Fig. S7). A study of the effect of the enantiopurity of ligand L1 on the enantioselectivity of the product 3aa demonstrated an obvious linear effect (see Supplementary Fig. S8). This observation suggested that the active catalyst species for the chiral induction in $\mathrm{C}-\mathrm{O}$ cross-coupling was consistent with a 1:1 ratio of the copper catalyst and chiral bisoxazoline ligand L1.

To gain some more insights into the mechanism, we then carried out a series of control experiments (Fig. 4). When commonly used radical scavengers, 2,2,6,6-tetramethyl-1-piperidinyloxy (TEMPO) and $\mathrm{PhSeSePh}$, were added to the model reaction of $1 \mathrm{a}$ and $\mathbf{2 a}$ under the standard conditions, the formation of desired product 3aa was completely inhibited in both cases. And the radical trapping adducts $\mathbf{1 2}$ and $\mathbf{1 3}$ could be detected by high-resolution mass spectrometry (HRMS) or isolated in quantitative yield (Fig. 4a). These results suggest the possible involvement of cyanoalkyl radical 2a-B that should be formed from the iminyl radical $\mathbf{2 a}-\mathbf{A}$ through its $\beta-\mathrm{C}-\mathrm{C}$ bond cleavage ${ }^{43}$. In addition, when the radical clock substrate $\mathbf{1 4}$ was reacted with $\mathbf{2 a}$, the cyclopropyl group was completely opened to give product $\mathbf{1 5}$ in modest yield with $83 \%$ ee (Fig. 4b). Thus, this radical clock experiment also provides further evidence of a radical process. To understand the behavior of the $O$-nucleophile-carboxylic anion-formed upon SET reduction of oxime ester, crossover experiments with two different oxime esters were performed (Fig. 4c). When equimolar amounts of oxime esters $\mathbf{2} \mathbf{h}$ and $\mathbf{5 a}$ were employed, four cross-coupling products were isolated with similar yields and enantioselectivity. In particular, the formation of formal three-component cross-coupled products 3aa and $\mathbf{1 6}$ implied that copper-catalyzed C-O bond-forming step should occur through out-of-cage coupling of radical intermediate and carboxylic anion. 
a Reactions in the presence of TEMPO or PhSeSePh
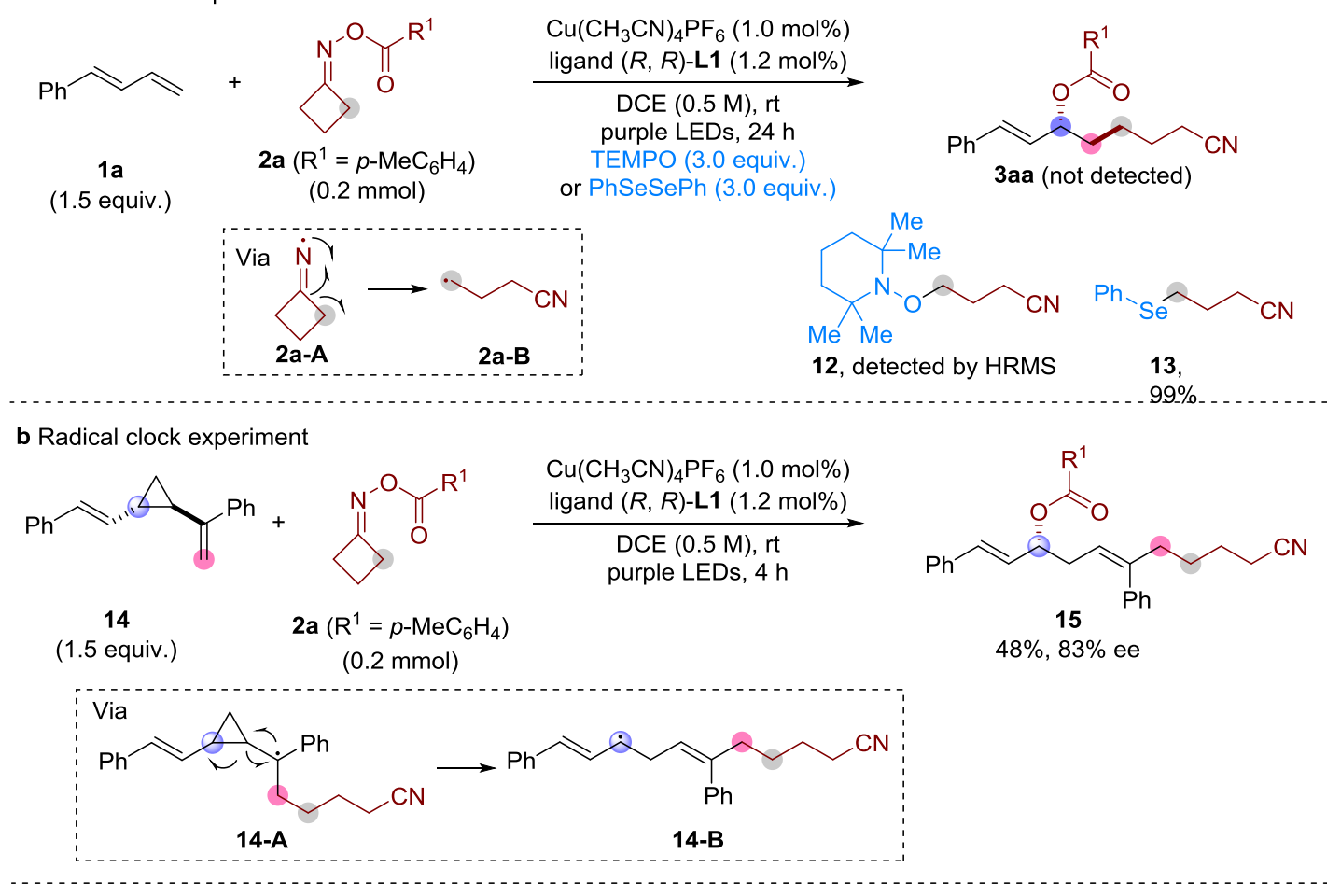

c Crossover reactions with two different oxime esters

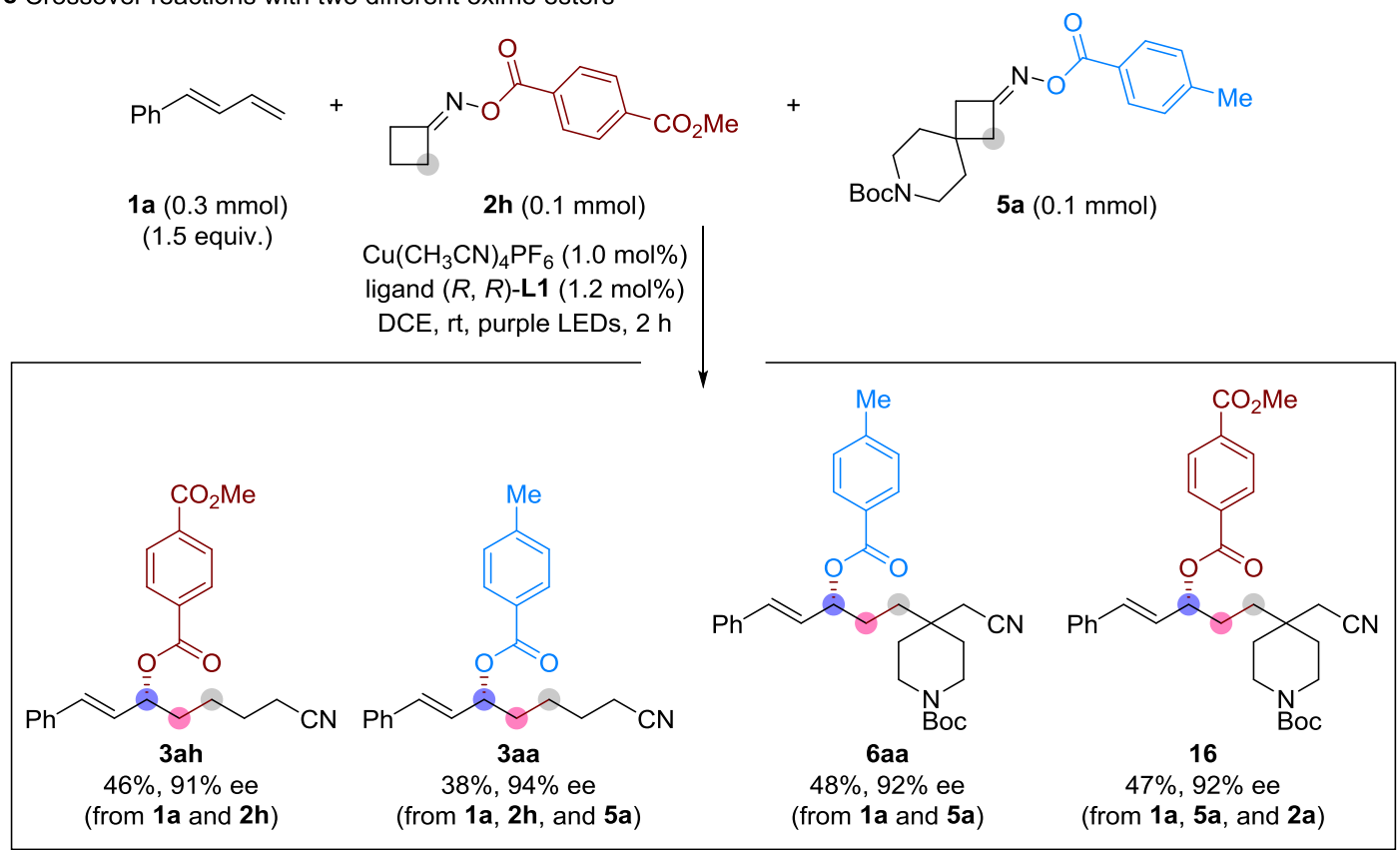

Fig. 4 | Mechanistic studies. a, Reactions in the presence of radical scavengers. b, Radical clock experiment. c, Crossover experiments with two different oxime esters.

To further obtain a deeper understanding of such photoinduced copper-catalyzed asymmetric C-O cross-coupling, several elementary steps involving regioselectivity and stereoselectivity were evaluated through density functional theory (DFT) calculations at the $\mathrm{SMD}^{46}(\mathrm{DCE}) / \mathrm{MO6}^{47} /\left[6-311++\mathrm{G}(\mathrm{d}, \mathrm{p}) / \mathrm{SDD}^{48}\right.$ $(\mathrm{Cu})] / / \mathrm{M06} /\left[6-31 \mathrm{G}(\mathrm{d}) / \mathrm{LanL} \mathrm{DZ} \mathrm{Z}^{49}(\mathrm{Cu})\right]$ level. According to our previous study ${ }^{43}$, the photoexcited state ${ }^{*} \mathrm{Cu}(\mathrm{I})$ complex can be oxidatively quenched by oxime ester (2a) via a SET process to generate a radical anion (2a') and a ground-state $\mathrm{Cu}(\mathrm{II})$ cation. As shown in Fig. $5 \mathrm{a}$, the resultant radical anion $\mathbf{2} \mathbf{a}^{\prime}$ undergoes 
three key elementary steps, $\mathrm{N}-\mathrm{O}$ bond cleavage, $\beta-\mathrm{C}-\mathrm{C}$ bond cleavage, and radical addition to $1 \mathrm{a}$, to provide the allyl radical (1a-A). TS1, TS2 and TS3 are the corresponding transition states for these three steps with the Gibbs activation energies $\left(\Delta G^{\circ \ddagger}\right)$ of $7.1,8.5$ and $5.1 \mathrm{kcal} \mathrm{mol}^{-1}$, respectively. In TS1, the N-O bond is elongated to $1.62 \AA$ from $1.39 \AA$ in $2 \mathrm{a}^{\prime}$, which indicates the breaking of $\mathrm{N}-\mathrm{O}$ bond is in progress. When the $\mathrm{N}-\mathrm{O}$ bond breaks, the spin densities of the resultant $\mathrm{N}-\mathrm{O}$ cleaved intermediate $\mathbf{2} \mathrm{a}$ " is mainly localized on $\mathrm{N}$ with 0.94 . Then, the iminyl radical (2a-A) is dissociated from $\mathbf{2 a "}$ " with a negative Gibbs free energy change $\left(\Delta G^{\circ}\right)$ of $-2.4 \mathrm{kcal} \mathrm{mol}^{-1}$. In TS2, $\beta-\mathrm{C}-\mathrm{C}$ bond of $2 \mathrm{a}-\mathrm{A}$ is activated to $1.99 \AA$ from $1.54 \AA$ in 2a-A, which means the cyanoalkyl radical (2a-B) is forming. When the $\beta-C-C$ bond is cleaved, the spin density becomes mainly localized on the terminal C4 of 2a-B with 1.04. In TS3, the C1'-C4 distance is shortened to $2.47 \AA$, which indicates the $\mathrm{C1}^{\prime}-\mathrm{C} 4$ bond is gradually forming. When 2a-B attacks the terminal position of 1-phenylbutadiene (1a), the spin densities become mainly localized on the $\mathrm{C2}^{\prime}$ and C4' of radical intermediate $1 \mathrm{a}-\mathrm{A}$ with 0.50 and 0.57 , respectively. Overall, the allyl radical formation occurs with a small energy barrier of $8.5 \mathrm{kcal} \mathrm{mol}^{-1}$ and a negative $\Delta G^{\circ}$ value of $-87.5 \mathrm{kcal} \mathrm{mol}^{-1}$. Subsequently, as shown in Fig. 5b, two possible $\mathrm{C}-\mathrm{O}$ bond-forming reductive eliminations have been investigated: one is the $\mathrm{C2}^{\prime}-\mathrm{O}$ reductive elimination from the stable $\pi$-allylcopper complex $\mathbf{D}-\mathbf{I}^{\mathbf{R}}$ to provide the 1,2 -adduct $(R)$-3aa (path a), whereas the other is the $C 4^{\prime}-O$ reductive elimination from $\pi$-benzylcopper complex $D$ - $I^{R}$ to obtain 1,4-adduct (R)-4aa (path b). $\mathbf{T S 4} \mathbf{a}^{\mathbf{R}}$ and $\mathbf{T S} \mathbf{S} \mathbf{b}^{\mathbf{R}}$ are the corresponding transition states for these two steps with the $\Delta G^{\circ \ddagger}$ values of 6.7 and $9.6 \mathrm{kcal} \mathrm{mol}^{-1}$, respectively. The $\mathrm{C2}^{\prime}-\mathrm{O}$ distance is shortened to $2.03 \AA$ in $\mathbf{T S} 4 a^{R}$ from $2.82 \AA$ in $\mathbf{D}-\mathbf{I}$, while the $\mathrm{C}^{\prime}-\mathrm{O}$ distance is shortened to $2.08 \AA$ in $\mathbf{T S}^{\prime} \mathbf{b}^{\mathbf{R}}$ from $3.08 \AA$ in $\mathrm{D}-\mathrm{II}^{\mathrm{R}}$, which indicates the bond formations of $\mathrm{C2}^{\prime}-\mathrm{O}$ and $\mathrm{C} 4^{\prime}-\mathrm{O}$ are in progress. The former path a occurs much more favorably by $2.9 \mathrm{kcal} / \mathrm{mol}$ than the latter path $\mathrm{b}$ due to the stable $\pi$-allylcopper interaction. Thus, the 1,2-adduct is a major product both in thermodynamics and kinetics, which is in line with the regioselectivtiy observed experimentally. In order to disclose the origin of high enantioselectivity, two favorable enantio-determining $\mathrm{C2}^{\prime}-\mathrm{O}$ reductive eliminations leading to $(R)$-3aa and $(S)$-3aa have been located, as shown in Fig. 5c. Starting from $\mathrm{Cu}(\mathrm{II})$ benzoate complex $\mathbf{C}$, the allyl radical 1a-A is electrophilically captured by the $\mathrm{Cu}(\mathrm{II})$ center from the Re-face and Si-face to generate $R$-type and $S$-type carbon-centered chiral $\mathrm{Cu}(\mathrm{III})$ intermediates $\mathbf{D}-\mathrm{I}^{\mathbf{R}}$ and $\mathbf{D}-\mathbf{I}^{\mathbf{S}}$, respectively. $\mathbf{D}-\mathrm{I}^{\mathbf{R}}$ is more stable than $\mathbf{D}-\mathbf{I}^{\mathbf{S}}$ by 5.1 $k c a l \mathrm{~mol}^{-1}$. Then, the $\mathrm{C2}^{\prime}-\mathrm{O}$ reductive eliminations of $\mathrm{D}-\mathrm{I}^{\mathrm{R}}$ and $\mathrm{D}-\mathrm{I}^{\mathrm{S}}$ occurs through $\mathrm{TS} 4 \mathrm{a}^{\mathrm{R}}$ and $\mathrm{TS} 4 \mathrm{a}^{\mathrm{S}}$ to provide the asymmetric $\mathrm{C}-\mathrm{O}$ cross-coupling products $(R)$-3aa and $(S)$-3aa. $\mathrm{TS}^{\mathrm{R}} \mathrm{a}^{\mathrm{R}}$ leading to $R$-configured product is lower than $\mathrm{TS}^{\mathrm{S}} \mathrm{a}^{\mathrm{S}}$ leading to $S$-configured product by $4.2 \mathrm{kcal} \mathrm{mol}^{-1}$, which is qualitatively in agreement with the observed $94 \%$ ee ((R)-3aa) (Fig. 5d). To understand well the reason why the $R$-configured product shows excellent enantioselectivity, further structural analysis from the perspective of the steric effect suggests that the steric hindrance between the aryl group of chiral ligand and the carboxylic group of substrate may be responsible for determining the enantioselectivity of $R$-configured product. 


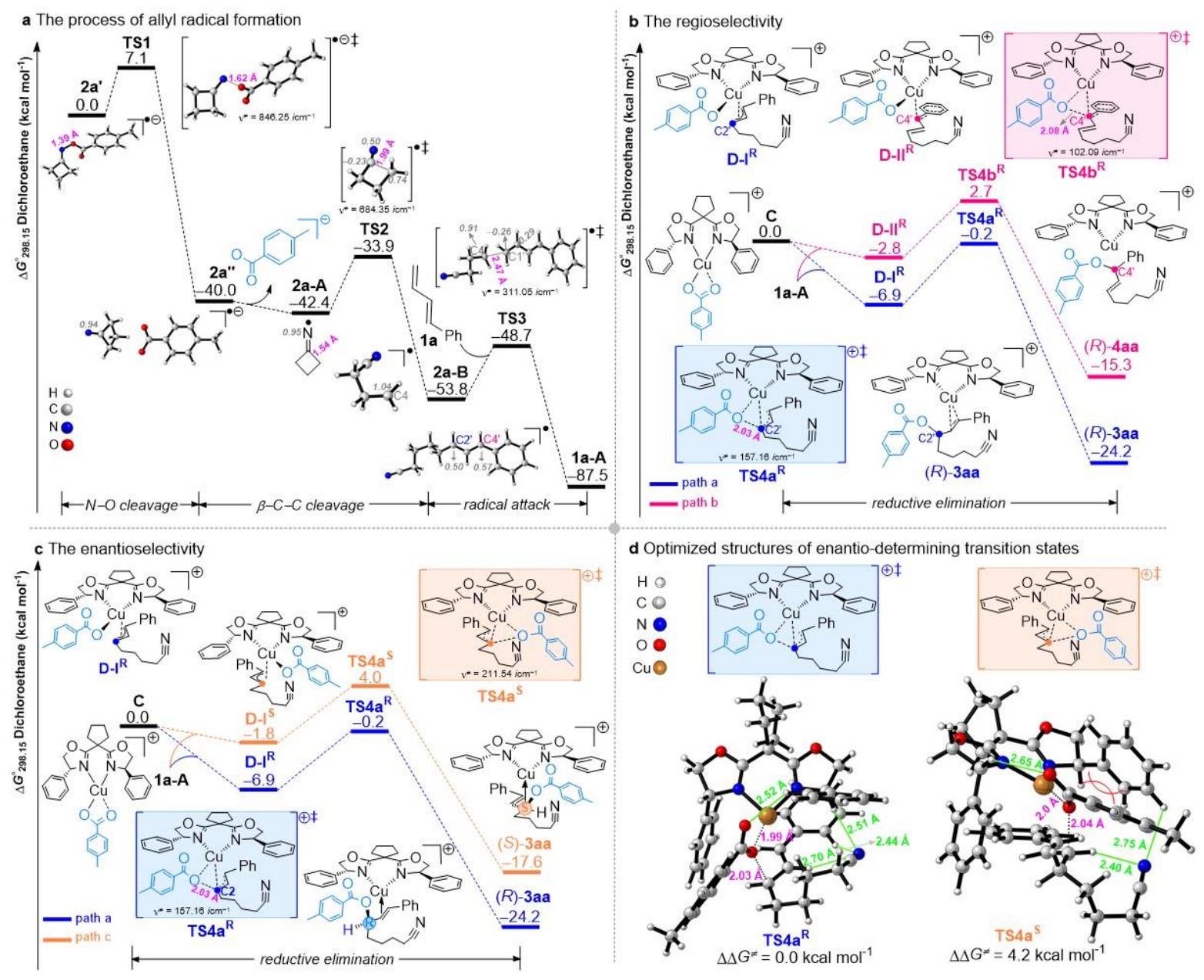

Fig. 5 | Results of density functional theory (DFT) calculations (all values are in kilocalories per mole). a, The process of allyl radical formation. $\mathbf{b}$, Formation of the allycopper complex and the regioselectivity of the $\mathrm{C}-\mathrm{O}$ cross-coupling reaction. $\mathbf{c}$, The enantioselectivity of the $\mathrm{C}-\mathrm{O}$ cross-coupling reaction. $\mathbf{d}$, Optimized structures of enantio-determining transition states.

To shed more light on the intermediacy of radical intermediates, EPR experiments were also performed (Fig. 6a). It was found that a solution of $2 a$ and $\mathbf{L} \mathbf{1} / \mathrm{Cu}(\mathrm{I})\left(\mathrm{CH}_{3} \mathrm{CN}\right)_{4} \mathrm{PF}_{6}$ in $\mathrm{DCE}$ show obvious $\mathrm{Cu}$ "l signals under visible light irradiation (Fig. 6a (i)). An iminyl radical trapping adduct 2a-A-I was also probed by adding radical trap 5,5-dimethyl-1-pyrroline-N-oxide (DMPO) (Fig. 6a (ii)). These results indicate the involvement of iminyl radical and its formation can only occur through a photoinduced copper(I)-catalyzed SET process.

Based on these mechanistic studies, we proposed a potential mechanism for this transformation with the model reaction as an example (Fig. 6b). First, irradiation of a $\mathbf{L} 1 \mathrm{Cu}(\mathrm{I}) \mathrm{X}$ complex (A) by purple light could lead to its excited state $[\mathbf{L C C u}(\mathbf{I}) \mathrm{X}]^{*}(\mathbf{B})$, which would then engage in a single electron transfer (SET) process with redox-active oxime ester $\mathbf{2 a}$ to generate an iminyl radical $\mathbf{2 a - A}$ and $\mathbf{L 1 C U}(\mathrm{CI})$ species with release of carboxylic anion $\left(\mathrm{R}^{1} \mathrm{CO}_{2}{ }^{-}\right)$. Meanwhile, coordination of the waste carboxylic anion $\left(\mathrm{R}^{1} \mathrm{CO}_{2}{ }^{-}\right)$ with the $\mathbf{L} \mathbf{C} \mathbf{C u}(\mathrm{II})$ species could result in a copper-nucleophile complex (C). Next, iminyl radical 2A-a undergoes facile $\beta-C-C$ bond cleavage to form cyanoalkyl radical $2 a-B$ that adds to the terminal position of diene $\mathbf{1 a}$ to furnish radical species $\mathbf{1 a - A}$. At this stage, radical $\mathbf{1 a - A}$ might associate with $\mathbf{C u}(\mathrm{II})$ complex $\mathbf{C}$ to form $\pi$-allyl $\mathrm{Cu}(\mathrm{III})$ complex $\mathbf{D}$ (specifically energetically favorable $\mathbf{D}-\mathrm{I}^{\mathbf{R}}$ as shown in Fig. 5$)^{50}$. However, 
$\pi$-allyl $\mathbf{C u}(\mathrm{III})$ complex $\mathbf{D}$ might exist in equilibrium with hybrid allylic $\mathrm{Cu}(\mathrm{II})$ radical, which cannot be ruled out at the current stage. Finally, $\mathrm{sp}^{3} \mathrm{C}-\mathrm{O}$ bond formation could occur enantioselectively to afford product 3aa and regenerates $\mathrm{LICU}(\mathrm{I}) \mathrm{X}$ complex (A).

a Electron paramagnetic resonance studies<smiles>[R]C(=O)ON=C1CCC1</smiles>

2a $\left(\mathrm{R}^{1}=p-\mathrm{MeC}_{6} \mathrm{H}_{4}\right)$
$\mathrm{Cu}\left(\mathrm{CH}_{3} \mathrm{CN}\right)_{4} \mathrm{PF}_{6}(5 \mathrm{~mol} \%)$

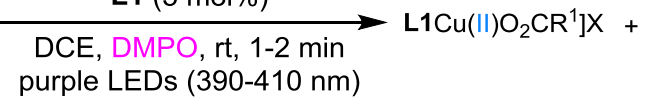
purple LEDs $(390-410 \mathrm{~nm})$

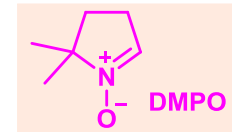

(i) irradiation for $2 \mathrm{~min}, 150 \mathrm{k}$

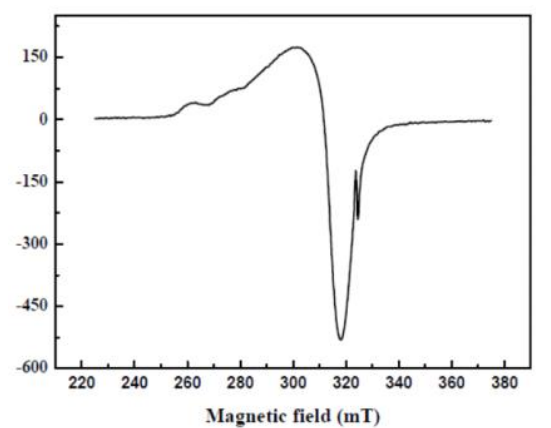

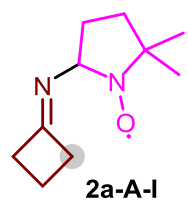

2a-A-I

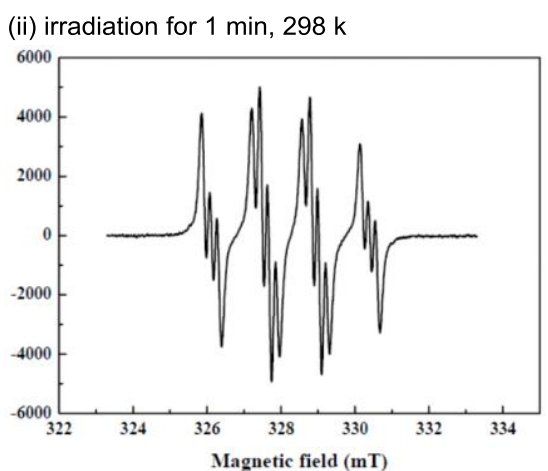

b Proposed mechanism

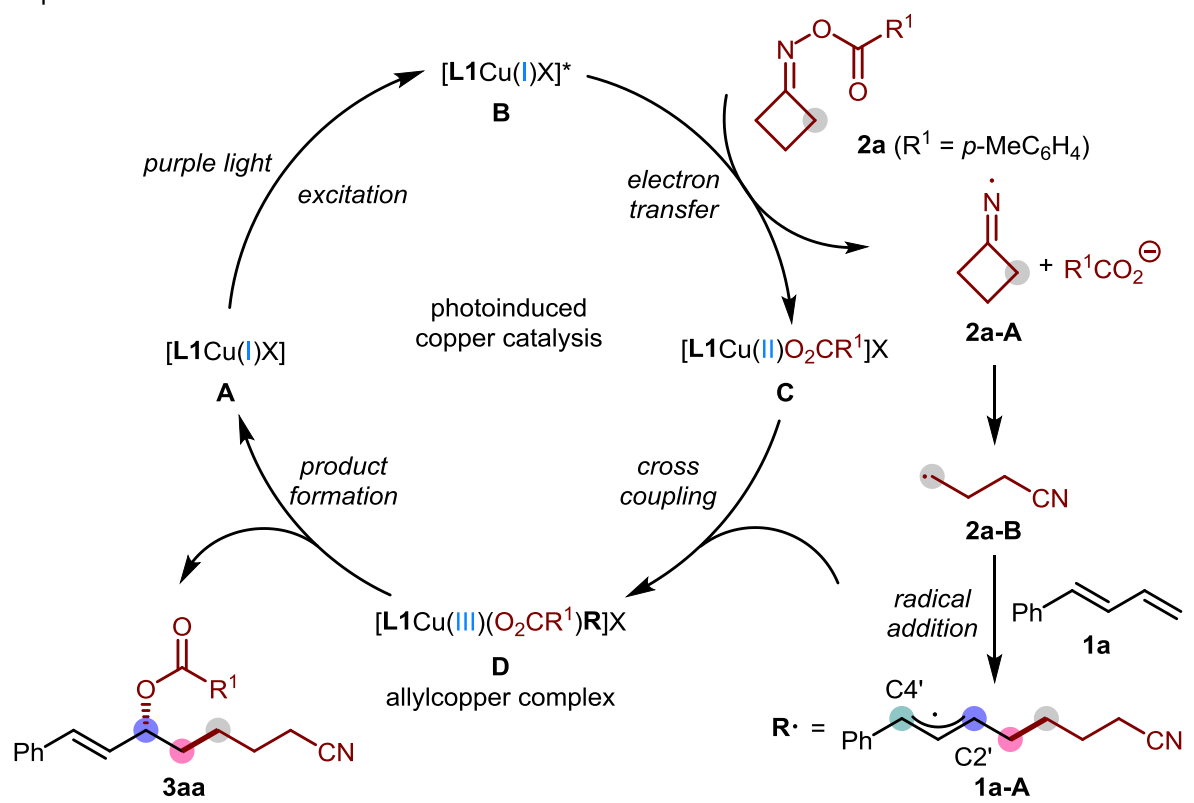

Fig. 6 | Electron paramagnetic resonance (EPR) studies and proposed mechanism. a, Electron paramagnetic resonance studies (X band, $9.045 \mathrm{GHz}$, g value 2.004). (i) EPR spectra of $\mathrm{Cu}$ (II); (ii) EPR spectra of spin adduct $\mathbf{2 a - A - I}$. b, Proposed catalytic cycle.

\section{Conclusion}

In conclusion, we have developed a photoinduced copper-catalyzed intermolecular asymmetric C-O cross-coupling that enables overall transformation of readily available oxime esters and 1,3-dienes to the corresponding diversely substituted chiral allylic esters with high regio- and enantioselectivity. This redox-neutral reaction proceeds at room temperature under visible light irradiation, and features the use 
of a copper-based chiral catalyst as both photoredox catalyst for radical generation and the source of asymmetric induction in $\mathrm{C}-\mathrm{O}$ cross-coupling. Key to the success of this transformation is the establishment of a general strategy for formation of $\pi$-allylcopper complex through a radical pathway under visible light irradiation. This strategy provides an alternative to the in situ-generation of $\pi$-allylcopper(III) complexes that are typically from nulceophilic organometallic reagents and pre-functionalized allylic systems. Overall, considering that photochemistry can generate various reactive intermediates that would otherwise be difficult to produce under thermal conditions ${ }^{51}$, many exciting extensions of this radical strategy to other related catalytic asymmetric cross-couplings can be envisaged.

\section{Methods}

General procedure for the synthesis of products 3 and 7 . In a flame-dried $10.0 \mathrm{~mL}$ Schlenk tube equipped with a magnetic stirrer bar was charged sequentially with $\mathrm{Cu}\left(\mathrm{CH}_{3} \mathrm{CN}_{4}{ }_{4} \mathrm{PF}_{6}(0.75 \mathrm{mg}, 0.002 \mathrm{mmol}, 1.0 \mathrm{~mol} \%)\right.$ and $\mathbf{L} \mathbf{1}(0.86 \mathrm{mg}, 0.0024 \mathrm{mmol}, 1.2 \mathrm{~mol} \%)$, followed by the addition of 1,2-dichloroethane $(4.0 \mathrm{~mL})$. Then the mixture was stirred at room temperature for $30 \mathrm{~min}$. To the resulting mixture were added $1(0.30$ mmol, 1.5 equiv.) and $2(0.20 \mathrm{mmol}, 1.0$ equiv.) under Ar. The mixture was stirred at a distance of $\sim 1 \mathrm{~cm}$ from two $20 \mathrm{~W}$ Kessil purple LEDs at rt for about 2-24 $\mathrm{h}$ until the reaction was completed, as monitored by TLC analysis. The residue was purified by acidic or neutral alumina chromatography with petroleum ether and ethyl acetate (PE/EA) $(10: 1, v / v)$ to afford 3 or 7.

Computational method. All density functional theory (DFT) calculations were carried out with the Gaussian 16 package. Molecular geometries of all studied systems were optimized at M06[6-31G(d)/LanL2DZ(Cu)] level. Frequency calculations were performed to confirm all the stationary points as local minimum (zero imaginary frequencies) or transition states (one imaginary frequency). Intrinsic reaction coordinate (IRC) calculations were conducted to verify that each transition state actually connects the two corresponding minimum. In addition, the single-point energies of all stationary points were performed at the M06[6-311++G(d,p)/SDD(Cu)] level. The single-point calculations were considered the solvent effects with the SMD model in 1,2-dichloroethane (DCE) solution.

Preprint. A previous version of this work was published as a preprint ${ }^{52}$.

\section{Data availability}

Data relating to the characterization data of materials and products, general methods, optimization studies, experimental procedures, mechanistic studies, mass spectrometry, high-performance liquid chromatography and NMR spectra, computational studies are available in the Supplementary Information.

\section{References}

1. Roughley, S. D. \& Jordan, A. M. The medicinal chemist's toolbox: An analysis of reactions used in the pursuit of drug candidates. J. Med. Chem. 54, 3451-3479 (2011).

2. Boström, J., Brown, D. G., Young, R. J. \& Keserü, G. M. Expanding the medicinal chemistry synthetic toolbox. Nat. Rev. Drug Disc. 17, 709-727 (2018).

3. Chemler, S. R. Copper's Contribution to amination catalysis. Science 341, 624-626 (2013).

4. Choi, J. \& Fu, G. C. Transition metal-catalyzed alkyl-alkyl bond formation: Another dimension in cross-coupling chemistry. Science 356, 152-159 (2017).

5. Bhunia, S., Pawar, G. G., Kumar, S. V., Jiang, Y. \& Ma, D. Selected copper-based reactions for C-N, C-O, 
C-S, and C-C bond formation. Angew. Chem. Int. Ed. 56, 16136-16179 (2017).

6. Cheng, L. J. \& Mankad, N. P. C-C and C-X coupling reactions of unactivated alkyl electrophiles using copper catalysis. Chem. Soc. Rev. 49, 8036-8064 (2020).

7. Kainz, Q. M. et al. Asymmetric copper-catalyzed C-N cross-couplings induced by visible light. Science 351, 681-684 (2016).

8. Bartoszewicz, A., Matier, C. D. \& Fu, G. C. Enantioconvergent alkylations of amines by alkyl electrophiles: Copper-catalyzed nucleophilic substitutions of racemic $\alpha$-halolactams by indoles. J. Am. Chem. Soc. 141, 14864-14869 (2019).

9. Lin, J.-S. et al. A Dual-catalytic strategy to direct asymmetric radical aminotrifluoromethylation of alkenes. J. Am. Chem. Soc. 138, 9357-9360 (2016).

10. Wang, F.-L. et al. Catalytic asymmetric radical diamination of alkenes. Chem 3, 979-990 (2017).

11. Lin, J.-S. et al. Catalytic asymmetric radical aminoperfluoroalkylation and aminodifluoromethylation of alkenes to versatile enantioenriched-fluoroalkyl amines. Nat. Commun. 8, 14841 (2017).

12. $\mathrm{Wu}$, L. et al. Anionic bisoxazoline ligands enable copper-catalyzed asymmetric radical azidation of acrylamides. Angew. Chem. Int. Ed. 60, 6997-7001 (2021).

13. Cecere, G., Konig, C. M., Alleva, J. L. \& MacMillan, D. W. Enantioselective direct $\alpha$-amination of aldehydes via a photoredox mechanism: a strategy for asymmetric amine fragment coupling. J. Am. Chem. Soc. 135, 11521-11524 (2013).

14. Hu, Y. et al. Enantioselective radical construction of 5-membered cyclic sulfonamides by metalloradical C-H amination. J. Am. Chem. Soc. 141, 18160-18169 (2019).

15. Nakafuku, K. M. et al. Enantioselective radical C-H amination for the synthesis of $\beta$-amino alcohols. Nat. Chem. 12, 697-704 (2020).

16. Zhu, R. \& Buchwald, S. L. Enantioselective functionalization of radical Intermediates in redox catalysis: Copper-catalyzed asymmetric oxytrifluoromethylation of alkenes. Angew. Chem. Int. Ed. 52, 12655-12658 (2013).

17. Zhu, R. \& Buchwald, S. L. Versatile enantioselective synthesis of functionalized lactones via copper-catalyzed radical oxyfunctionalization of alkenes. J. Am. Chem. Soc. 137, 8069-8077 (2015).

18. Li, X.-T., Gu, Q.-S., Dong, X.-Y., Meng, X. \& Liu, X.-Y. A copper catalyst with a cinchona-alkaloid-based sulfonamide ligand for asymmetric radical oxytrifluoromethylation of alkenyl oximes. Angew. Chem. Int. Ed. 57, 7668-7672 (2018).

19. Zhang, G. et al. Asymmetric coupling of carbon-centered radicals adjacent to nitrogen: copper-catalyzed cyanation and etherification of enamides. Angew. Chem. Int. Ed. 59, 20439-20444 (2020).

20. $\mathrm{Hu}, \mathrm{H}$. et al. Copper-catalysed benzylic $\mathrm{C}-\mathrm{H}$ coupling with alcohols via radical relay enabled by redox buffering. Nat. Catal. 3, 358-367 (2020).

21. Alexakis, A., Backvall, J. E., Krause, N., Pamies, O. \& Dieguez, M. Enantioselective copper-catalyzed conjugate addition and allylic substitution reactions. Chem. Rev. 108, 2796-2823 (2008).

22. Susse, L. \& Stoltz, B. M. Enantioselective formation of quaternary centers by allylic alkylation with first-row transition-metal catalysts. Chem. Rev. 121, 4084-4099 (2021).

23. Yoshikai, N. \& Nakamura, E. Mechanisms of nucleophilic organocopper(I) reactions. Chem. Rev. 112, 2339-2372 (2012).

24. Hossain, A., Bhattacharyya, A. \& Reiser, O. Copper's rapid ascent in visible-light photoredox catalysis. Science 364, 450-461 (2019).

25. Wang, F., Chen, P. \& Liu, G. Copper-catalyzed radical relay for asymmetric radical transformations. Acc. 
Chem. Res. 51, 2036-2046 (2018).

26. Li, Z.-L., Fang, G.-C., Gu, Q.-S. \& Liu, X.-Y. Recent advances in copper-catalysed radical-involved asymmetric 1,2-difunctionalization of alkenes. Chem. Soc. Rev. 49, 32-48 (2020).

27. $\mathrm{Li}$, J. et al. Site-specific allylic $\mathrm{C}-\mathrm{H}$ bond functionalization with a copper-bound $\mathrm{N}$-centred radical. Nature 574, 516-521 (2019).

28. Huang, H.-M. et al. Catalytic radical generation of $\pi$-allylpalladium complexes. Nat. Catal. 3, 393-400 (2020).

29. Huang, H. M. et al. Three-component, interrupted radical Heck/allylic substitution cascade involving unactivated alkyl bromides. J. Am. Chem. Soc. 142, 10173-10183 (2020).

30. Bellotti, P., Koy, M., Gutheil, C., Heuvel, S. \& Glorius, F. Three-component three-bond forming cascade via palladium photoredox catalysis. Chem. Sci. 12, 1810-1817 (2021).

31. Patra, T., Das, M., Daniliuc, C. G. \& Glorius, F. Metal-free photosensitized oxyimination of unactivated alkenes with bifunctional oxime carbonates. Nat. Catal. 4, 54-61 (2021).

32. Schwarz, J. L., Huang, H.-M., Paulisch, T. O. \& Glorius, F. Dialkylation of 1,3-dienes by dual photoredox and chromium catalysis. ACS Catal. 10, 1621-1627 (2020).

33. Shing Cheung, K. P., Kurandina, D., Yata, T. \& Gevorgyan, V. Photoinduced palladium-catalyzed carbofunctionalization of conjugated dienes proceeding via radical-polar crossover scenario: 1,2-aminoalkylation and beyond. J. Am. Chem. Soc. 142, 9932-9937 (2020).

34. Li, F. et al. Photocatalytic generation of $\pi$-allyltitanium complexes via radical intermediates. Angew. Chem. Int. Ed. 60, 1561-1566 (2021).

35. Yu, X.-Y., Zhao, Q.-Q., Chen, J., Xiao, W.-J. \& Chen, J.-R. When light meets nitrogen-centered radicals: From reagents to catalysts. Acc. Chem. Res. 53, 1066-1083 (2020).

36. Hickman, A. J. \& Sanford, M. S. High-valent organometallic copper and palladium in catalysis. Nature 484, 177-185 (2012).

37. Casitas, A. \& Ribas, X. The role of organometallic copper(iii) complexes in homogeneous catalysis. Chem. Sci. 4, 2301 (2013).

38. Li, G., Huo, X., Jiang, X. \& Zhang, W. Asymmetric synthesis of allylic compounds via hydrofunctionalisation and difunctionalisation of dienes, allenes, and alkynes. Chem. Soc. Rev. 49, 2060-2118 (2020).

39. Huang, H.-M., Bellotti, P. \& Glorius, F. Transition metal-catalysed allylic functionalization reactions involving radicals. Chem. Soc. Rev. 49, 6186-6197 (2020).

40. Du, H., Zhao, B., Yuan, W. \& Shi, Y. Cu(I)-catalyzed asymmetric diamination of conjugated dienes. Org. Lett. 10, 4231-4234 (2008).

41. Xiao, F., Guo, Y. \& Zeng, Y.-F. Recent developments in radical cross-coupling of redox-active cycloketone oximes. Adv. Synth. Catal. 363, 120-143 (2021).

42. Zhao, B. \& Shi, Z. Copper-catalyzed intermolecular Heck-like coupling of cyclobutanone oximes initiated by selective C-C bond cleavage. Angew. Chem. Int. Ed. 56, 12727-12731 (2017).

43. Yu, X.-Y., Zhao, Q.-Q., Chen, J., Chen, J.-R. \& Xiao, W.-J. Copper-catalyzed radical cross-coupling of redox-active oxime esters, styrenes and boronic acids. Angew. Chem. Int. Ed. 57, 15505-15509 (2018).

44. Xiong, Y., Sun, Y. \& Zhang, G. Recent advances on catalytic asymmetric difunctionalization of 1,3-dienes. Tetrahedron Lett. 59, 347-355 (2018).

45. Wu, X. \& Gong, L.-Z. Palladium(0)-catalyzed difunctionalization of 1,3-dienes: From racemic to enantioselective. Synthesis 51, 122-134 (2019).

46. Marenich, A. V., Cramer, C. J. \& Truhlar, D. G. Universal solvation model based on solute electron 
density and on a continuum model of the solvent defined by the bulk dielectric constant and atomic surface tensions. J. Phys. Chem. B 113, 6378-6396 (2009).

47. Zhao, Y. \& Truhlar, D. G. The M06 suite of density functionals for main group thermochemistry, thermochemical kinetics, noncovalent interactions, excited states, and transition elements: two new functionals and systematic testing of four M06-class functionals and 12 other functionals. Theor. Chem. Acc. 120, 215-241 (2008).

48. Dolg, M., Wedig, U., Stoll, H. \& Preuss, H. Energy-adjusted abinitio pseudopotentials for the first row transition elements. J. Chem. Phys. 86, 866-872 (1987).

49. Hay, P. J. \& Wadt, W. R. Ab initio effective core potentials for molecular calculations. potentials for $\mathrm{K}$ to Au including the outermost core orbitals. J. Chem. Phys. 82, 299-310 (1985).

50. Eames, J. \& Watkinson, M. Catalytic allylic oxidation of alkenes using an asymmetric Kharasch-Sosnovsky reaction. Angew. Chem. Int. Ed. 40, 3567-3571 (2001).

51. Albini, A. \& Fagnoni, M. Photochemically-Generated Intermediates in Synthesis. (John Wiley \& Sons, 2013).

52. Chen, J. et al. Photoinduced copper-catalyzed asymmetric C-O cross-coupling. Preprint at ChemRxiv

\section{Acknowledgements}

We are grateful to the financial support from the National Natural Science Foundation of China (Nos. 21971081, 91856119, 21772053, 21820102003, 91956201, and 21773025), the Program of Introducing Talents of Discipline to Universities of China (111 Program, B17019), and the Excellent Doctoral Dissertation Cultivation Grant to J.C. and P.Z.W. from CCNU. We also thank Dr. Min Jiang (Hangzhou Normal University) for help with the EPR experiments. This paper is dedicated to Professor Ilhyong Ryu on the occasion of his 70th birthday.

\section{Author contributions}

J.C., P.-Z.W., G.Q.L., H.Q., B.Z., and X.-D.H. are responsible for the plan and implementation of the experimental work. Y.J.L and W.G. are responsible for implementation of the DFT calculations and related writing. J.-R.C. and W.-J.X. supervised the project and wrote the manuscript with revisions provided by the other authors. All authors discussed the results and commented on the manuscript.

\section{Competing interests}

The authors declare no competing interests. 